\title{
SPECTRAL PROBLEMS OF NON-SELF-ADJOINT $q$-STURM- LIOUVILLE OPERATORS IN LIMIT-POINT CASE
}

\author{
Bilender P. AllahVerdiev
}

\begin{abstract}
In this study, dissipative singular $q$-Sturm-Liouville operators are studied in the Hilbert space $\mathscr{L}_{r, q}^{2}\left(\mathbf{R}_{q,+}\right)$, that the extensions of a minimal symmetric operator in limitpoint case. We construct a self-adjoint dilation of the dissipative operator together with its incoming and outgoing spectral representations so that we can determine the scattering function of the dilation as stated in the scheme of Lax-Phillips. Then, we create a functional model of the maximal dissipative operator via the incoming spectral representation and define its characteristic function in terms of the Weyl-Titchmarsh function (or scattering function of the dilation) of a self-adjoint $q$-Sturm-Liouville operator. Finally, we prove the theorem on completeness of the system of eigenfunctions and associated functions (or root functions) of the dissipative $q$-Sturm-Liouville operator.
\end{abstract}

\section{Introduction and notations}

In this section, we introduce some of the needed $q$-notations and results (see $[3-6,9,10])$. Throughout this paper, $q$ is a positive number with $0<q<1$. For $\mu \in \mathbf{R}:=(-\infty, \infty)$, a set $A \subseteq \mathbf{R}$ is called a $\mu$-geometric set if $\mu t \in A$ for all $t \in A$. If $A \subseteq \mathbf{R}$ is a $\mu$-geometric set, then it contains all geometric sequences $\left\{\mu^{n} t\right\} \quad(n=0,1,2 \ldots), t \in A$. Let $f$ be a function, real or complex-valued, defined on a $q$-geometric set $A$. The $q$-difference operator is defined by

$$
D_{q} f(t):=\frac{f(t)-f(q t)}{t-q t}, \quad t \in A \backslash\{0\} .
$$

If $0 \in A$, the $q$-derivative at zero is defined by

$$
D_{q} f(0):=\lim _{n \rightarrow \infty} \frac{f\left(q^{n} t\right)-f(0)}{q^{n} t}
$$

2010 Mathematics Subject Classification. Primary 39A13, Secondary 33D05, 34B05, 34B24, 47B25, 47B44, 47A20, 47A40.

Key words and phrases. $q$-Sturm-Liouville equations; dissipative operators; self-adjoint dilation; scattering function; characteristic function; completeness of the root functions.

Received June 26, 2014; revised May 26, 2015. 
if the limit exists and does not depend on $t$. Since the formulation of the extension problems requires the definition of $D_{q^{-1}}$ in a same manner to be

$$
D_{q^{-1}} f(t):= \begin{cases}\frac{f(t)-f\left(q^{-1} t\right)}{t-q^{-1} t}, & t \in A \backslash\{0\}, \\ D_{q} f(0), & t=0,\end{cases}
$$

provided that $D_{q} f(0)$ exists. As a converse of the $q$-difference operator, Jackson's $q$-integration [12] is given by

$$
\int_{0}^{t} f(t) d_{q} \xi:=t(1-q) \sum_{n=0}^{\infty} q^{n} f\left(q^{n} t\right), \quad t \in A,
$$

provided that the series converges, and

$$
\int_{a}^{b} f(t) d_{q} t:=\int_{0}^{b} f(t) d_{q} t-\int_{0}^{a} f(t) d_{q} t, \quad a, b \in A .
$$

When required $q$ will be replaced by $q^{-1}$. The following facts, which will be used often, can be verified directly from the definition and will be used often:

$$
D_{q^{-1}} f(t)=\left(D_{q} f\right)\left(q^{-1} t\right), \quad D_{q}^{2} f\left(q^{-1} t\right)=q D_{q}\left[D_{q} f\left(q^{-1} t\right)\right]=D_{q^{-1}} D_{q} f(t) .
$$

Associated with this operator there is a non-symmetric formula for the $q$-differentation of a product

$$
D_{q}[f(t) g(t)]=f(q t) D_{q} g(t)+g(t) D_{q} f(t) .
$$

Through the remainder of the text, we deal only with functions $q$-regular at zero, that is, functions satisfying

$$
\lim _{n \rightarrow \infty} f\left(q^{n} t\right)=f(0) .
$$

The class of the functions which are $q$-regular at zero includes the continuous functions. If $f$ and $g$ are $q$-regular at zero, there is a rule of $q$-integration by parts given by

$$
\int_{0}^{a} g(t) D_{q} f(t) d_{q} t=(f g)(a)-(f g)(0)-\int_{0}^{a} D_{q} g(t) f(q t) d_{q} t .
$$

In [11], Hahn defined the $q$-integration for a function $f$ over $[0, \infty)$ by

$$
\int_{0}^{\infty} f(t) d_{q} t:=(1-q) \sum_{n=-\infty}^{\infty} q^{n} f\left(q^{n}\right)
$$

The $q$-difference calculus or quantum calculus was initiated in the beginning of the 19th century. The subject of $q$-differential equations has evolved into a multidisciplinary subject (see $[3,9,10]$ ). There are several physical models involving $q$-derivatives, $q$-integrals, $q$-exponential function, $q$-trigonometric func- 
tion, $q$-Taylor formula, $q$-Beta and $q$-Gamma functions, Euler-Maclaurin formula and their related problems (see $[3,9,10])$.

In [6], Annaby and Mansour studied a $q$-Sturm-Liouville eigenvalue problem and formulated a self-adjoint $q$-Sturm-Liouville operator in a Hilbert space. They also discussed properties of the eigenvalues and the eigenfunctions. Annaby et al. [4, 5] established the $q$-Titchmarsh-Weyl theory for singular $q$-Sturm-Liouville problems and defined $q$-limit-point and $q$-limit-circle singularities and gave sufficient conditions which guarantee that the singular point is in a limit-point case in [5].

Let $\mathscr{L}^{2}(\mathbf{R})$ be the Hilbert space consisting of all complex-valued functions $y$ such that

$$
\int_{-\infty}^{\infty}|y(t)|^{2} d t<\infty
$$

and with the inner product

$$
(y, z)=\int_{-\infty}^{\infty} y(t) \overline{z(t)} d t .
$$

We denote by $\mathscr{L}^{2}\left(\mathbf{R}_{-}\right)\left(\mathscr{L}^{2}\left(\mathbf{R}_{+}\right)\right)$, where $\mathbf{R}_{-}:=(-\infty, 0]\left(\mathbf{R}_{+}:=[0, \infty)\right)$, the Hilbert space consisting of all complex-valued functions $y$ satisfying

$$
\int_{-\infty}^{0}|y(t)|^{2} d t<\infty \quad\left(\int_{0}^{\infty}|y(t)|^{2} d t<\infty\right)
$$

and with the inner product

$$
(y, z)=\int_{-\infty}^{0} y(t) \overline{z(t)} d t \quad\left((y, z)=\int_{0}^{\infty} y(t) \overline{z(t)} d t\right) .
$$

Let $\mathscr{W}_{2}^{1}\left(\mathbf{R}_{ \pm}\right)$be the Sobolev space consisting of all functions $f \in \mathscr{L}^{2}\left(\mathbf{R}_{ \pm}\right)$ such that $f$ are locally absolutely continuous functions on $\mathbf{R}_{+}$and $f^{\prime} \in \mathscr{L}^{2}\left(\mathbf{R}_{+}\right)$.

We shall remind that the linear operator $\mathbf{T}$ (with dense domain $\operatorname{Dom}(\mathbf{T})$ ) acting on some Hilbert space $\mathbf{H}$ is called dissipative (accumulative) if $\operatorname{Im}(\mathbf{T} f, f) \geq 0(\operatorname{Im}(\mathbf{T} f, f) \leq 0)$ for all $f \in \operatorname{Dom}(\mathbf{T})([1,2,13,15,17,18])$.

It is better to recall that a linear operator $\mathbf{B}$ (with domain $\operatorname{Dom}(\mathbf{B})$ ) acting in a Hilbert space $\mathbf{H}$ is called completely non-self-adjoint (or pure) if the invariant subspace $\mathbf{M} \subseteq \operatorname{Dom}(\mathbf{B})(\mathbf{M} \neq\{0\})$ of the operator $\mathbf{B}$ whose restriction to $\mathbf{M}$ is self-adjoint, does not exist $([2,13,17])$.

Let $\Theta$ be an arbitrary non-constant inner function $([1,2,13,15,17])$ defined on the upper half-plane (we recall that a function $\Theta$ analytic in the upper halfplane $\mathbf{C}_{+}$is called inner function on $\mathbf{C}_{+}$if $|\Theta(\lambda)| \leq 1$ for $\lambda \in \mathbf{C}_{+}$, and $|\Theta(\lambda)|=1$ for almost all $\lambda \in \mathbf{R})$. Here and below, $\mathscr{H}_{+}^{2}$ denote the Hardy classes $([15,17])$ in $\mathscr{L}^{2}(\mathbf{R})$ consisting of the functions analytically extendable to the upper and lower half-planes, respectively. Let us consider the nontrivial subspace $\mathscr{K}=$ $\mathscr{H}_{+}^{2} \ominus \Theta \mathscr{H}_{+}^{2}$. The semigroup of the operators $\mathscr{X}(s)(s \geq 0), \mathscr{X}(s) \chi=\mathscr{P}\left[e^{i \lambda s} \chi\right]$, $\chi:=\chi(\lambda) \in \mathscr{K}$, where $\mathscr{P}$ is the orthogonal projection from $\mathscr{H}_{+}^{2}$ onto $\mathscr{K}$, acts in 
the subspace $\mathscr{K}$. The generator of the semigroup $\{\mathscr{X}(s)\}$ is denoted by $\mathscr{T}$ :

$$
\mathscr{T} \chi=\lim _{s \rightarrow+0}\left[(i s)^{-1}(\mathscr{X}(s) \chi-\chi)\right]
$$

which is a dissipative operator acting in $\mathscr{K}$ and having domain $\operatorname{Dom}(\mathscr{T})$ which consists of all vectors $\chi \in \mathscr{K}$ such that the limit exists. The operator $\mathscr{T}$ is called a model dissipative operator. This model dissipative operator, which is associated with the names of Lax and Phillips [14], is a special case of a more general model dissipative operator constructed by Sz.-Nagy and Foiaş [15]. The basic assertion is that $\Theta$ is the characteristic function of the operator $\mathscr{T}([1,2,13$, $15,17,18])$.

Let $\mathbf{S}$ denote the linear operator acting in the Hilbert space $\mathbf{H}$ with the domain $\operatorname{Dom}(\mathbf{S})$. We know that a complex number $\lambda_{0}$ is called an eigenvalue of an operator $\mathbf{S}$ if there exists a non-zero vector $z_{0} \in \operatorname{Dom}(\mathbf{S})$ satisfying the equation $\mathbf{S} z_{0}=\lambda_{0} z_{0}$; here, $z_{0}$ is called an eigenvector of $\mathbf{S}$ for $\lambda_{0}$. The eigenvector for $\lambda_{0}$ spans a subspace of $\operatorname{Dom}(\mathbf{S})$, called the eigenspace for $\lambda_{0}$ and the geometric multiplicity of $\lambda_{0}$ is the dimension of its eigenspace. The vectors $z_{1}, z_{2}, \ldots, z_{k}$ are called the associated vectors of the eigenvector $z_{0}$ if they belong to $\operatorname{Dom}(\mathbf{S})$ and $\mathbf{S} z_{j}=\lambda_{0} z_{j}+z_{j-1}, j=1,2, \ldots, k$. The element $z \in \operatorname{Dom}(\mathbf{S}), z \neq 0$ is called a root vector of the operator $A$ corresponding to the eigenvalue $\lambda_{0}$, if all powers of $\mathbf{S}$ are defined on this element and $\left(\mathbf{S}-\lambda_{0} I\right)^{m} z=0$ for some integer $m$. The set of all root vectors of $\mathbf{S}$ corresponding to the same eigenvalue $\lambda_{0}$ with the vector $z=0$ forms a linear set $N_{\lambda_{0}}$ and is called the root lineal. The dimension of the lineal $\mathbf{N}_{\lambda_{0}}$ is called the algebraic multiplicity of the eigenvalue $\lambda_{0}$. The root lineal $\mathbf{N}_{\lambda_{0}}$ coincides with the linear span of all eigenvectors and associated vectors of $\mathbf{S}$ corresponding to the eigenvalue $\lambda_{0}$. As a result, the completeness of the system of all eigenvectors and associated vectors of $\mathbf{S}$ is equivalent to the completeness of the system of all root vectors of this operator $([2,8,16])$.

Dissipative operators are one of the important classes of non-self-adjoint operators. It is well recognized that $[1,2,13,15,17,18]$ the theory of dilations with application of functional models gives an ample approach to the spectral theory of dissipative (contractive) operators. By carrying the complete information on the spectral properties of the dissipative operator, we can say that characteristic function plays the primary role in this theory. The spectral analysis of the dissipative operators can be studied with the help of the characteristic function. Using the theory of Sz.-Nagy-Foiaş, the dissipative operator can be handled as the model operator $[1,2,13,15,17,18]$. In the centre of this method, there is an information on the spectral properties of the dissipative operator. For example, the factorization of the characteristic function may help us about learning that whether the system of all eigenvectors and associated vectors is complete or not. To construct the characteristic function directly is quite hard. However, according to the results of Lax-Phillips, this construction can be done with the self-adjoint dilation and scattering function (see [14]). Efficiency of this approach for dissipative Schrödinger and Sturm-Liouville operators has been demonstrated, for example, in $[1,2,17,18]$. 
Let $\mathscr{L}_{r, q}^{2}\left(\mathbf{R}_{q,+}\right)$ (where $\mathbf{R}_{q,+}:=\left\{q^{n} \mid n \in \mathbf{Z}\right\}, \mathbf{Z}:=\{0, \pm 1, \pm 2, \ldots\}$ ) denote the Hilbert space (see [3]) consisting of all complex-valued functions $x$ defined on $\mathbf{R}_{q,+}$ satisfying

$$
\int_{0}^{\infty} r(t)|x(t)|^{2} d_{q} t<\infty
$$

and with the inner product

$$
(x, y)=\int_{0}^{\infty} r(t) x(t) \overline{y(t)} d_{q} t .
$$

where $r$ is a real-valued function defined on $\mathbf{R}_{q,+}$ and $r(t)>0$ for all $t \in \mathbf{R}_{q,+}$.

In this paper, we consider the dissipative singular $q$-Sturm-Liouville operators acting in the Hilbert space $\mathscr{L}_{r, q}^{2}\left(\mathbf{R}_{q,+}\right)$, that the extensions of a minimal symmetric operator with deficiency indices $(1,1)$ (in limit-point case). At first a self-adjoint dilation of the maximal dissipative operator is constructed and then its incoming and outgoing spectral representations are prescribed. These constructions will allow us to determine the scattering function of the dilation in terms of the Weyl-Titchmarsh function of self-adjoint operator according to the scheme of Lax and Phillips [14]. With the help of the incoming spectral representation, we construct a functional model of the maximal dissipative operator and determine its characteristic function in terms of the scattering function of self-adjoint dilation (or Weyl-Titchmarsh function of a self-adjoint $q$-Sturm-Liouville operator). Finally, using the results found for characteristic functions, we prove the theorem on completeness of the system of eigenfunctions and associated functions (or root functions) of the dissipative $q$-Sturm-Liouville operator.

\section{Dissipative $q$-Sturm-Liouville operator and self-adjoint dilation of the dissipative operator}

We consider the singular $q$-Sturm-Liouville expression $L$ as

$$
(L x)(t)=\frac{1}{r(t)}\left[-\frac{1}{q} D_{q^{-1}}\left(p(t) D_{q} x(t)\right)+v(t) x(t)\right], \quad t \in \mathbf{R}_{q,+},
$$

where $r, p$ and $v$ are real-valued functions defined on $\mathbf{R}_{q,+}$ and are $q$-regular at zero such that $p(t) \neq 0, r(t)>0$ for all $t \in \mathbf{R}_{q,+}$, and $D_{q}$ is the $q$-difference operator defined in (1.1).

Let us consider the linear set $\mathfrak{D}_{\max }$ consisting of all vectors $x \in \mathscr{L}_{r, q}^{2}\left(\mathbf{R}_{q,+}\right)$ such that $x$ and $\left(p D_{q}\right) x$ are $q$-regular at zero and $L x \in \mathscr{L}_{r, q}^{2}\left(\mathbf{R}_{+}\right)$. We define the maximal operator $\mathscr{L}_{\max }$ on $\mathfrak{D}_{\max }$ by the equality $\mathscr{L}_{\max } x=L x$.

For each $x, y \in \mathfrak{D}_{\max }$ we define the $q$-Wronski determinant (or $q$-Wronskian) as follows:

$$
\mathscr{W}_{q}[x, y](t)=x(t) D_{q} y(t)-D_{q} x(t) y(t), \quad t \in \mathbf{R}_{q,+} .
$$


For arbitrary $x, y \in \mathfrak{D}_{\max }, q$-Green's formula (or $q$-Lagrange's identity) is given by $([3,6])$

$$
\int_{0}^{t}(L x)(\xi) \overline{y(\xi)} d_{q} \xi-\int_{0}^{t} x(\xi) \overline{(L y)(\xi)} d_{q} \xi=[x, y](t)-[x, y](0), \quad t \in \mathbf{R}_{q,+},
$$

where $[x, y](t)$ denotes the $q$-Lagrange bracket defined by

$$
[x, y](t):=p(t)\left[x(t) \overline{D_{q^{-1}} y(t)}-D_{q^{-1}} x(t) \overline{y(t)}\right], \quad t \in \mathbf{R}_{q,+} .
$$

It is clear from (2.2) that limit

$$
[x, y](\infty):=\lim _{n \rightarrow \infty}[x, y]\left(q^{-n}\right)
$$

exists and is finite for all $x, y \in \mathfrak{D}_{\max }$. For any function $x \in \mathfrak{D}_{\max }, x(0)$ and $\left(p D_{q^{-1}} x\right)(0)$ can be defined by

$$
x(0):=\lim _{n \rightarrow \infty} x\left(q^{n}\right)
$$

and

$$
\left(p D_{q^{-1}} x\right)(0):=\lim _{n \rightarrow \infty}\left(p D_{q^{-1}} x\right)\left(q^{n}\right) .
$$

These limits exist and are finite (since $x$ and $\left(p D_{q^{-1}}\right) x$ are $q$-regular at zero). Let $\mathfrak{D}_{\min }$ be the linear set of all vectors $x \in \mathfrak{D}_{\max }$ satisfying the conditions

$$
x(0)=\left(p D_{q^{-1}} x\right)(0)=0, \quad[x, y](\infty)=0,
$$

for arbitrary $y \in \mathfrak{D}_{\max }$. The operator $\mathscr{L}_{\min }$, that is the restriction of the operator $\mathscr{L}_{\max }$ to $\mathfrak{D}_{\min }$ is called the minimal operator and the equalities $\mathscr{L}_{\max }=\mathscr{L}_{\min }^{*}$ holds. Further (it follows from $(2.3)) \mathscr{L}_{\min }$ is a closed symmetric operator with deficiency indices $(1,1)$ or $(2,2)([4,5,8,16])$.

In this paper, we assume that symmetric operator $\mathscr{L}_{\min }$ has deficiency indices $(1,1)$, so the case of limit-point occurs for expression $L$ or $\mathscr{L}_{\min }([4,5,7,8,16])$.

Let us consider the operator $T_{\alpha}$ with domain $\operatorname{Dom}\left(T_{\alpha}\right)$ consisting of vectors $x \in \mathfrak{D}_{\max }$ which satisfy the boundary conditions

$$
\left(p D_{q^{-1}}\right) x(0)-\alpha x(0)=0, \quad \operatorname{Im} \alpha>0 .
$$

THEOREM 2.1. The operator $T_{\alpha}$ is dissipative in space $\mathscr{L}_{r, q}^{2}\left(\mathbf{R}_{q,+}\right)$.

Proof. Let $x \in \operatorname{Dom}\left(T_{\alpha}\right)$. Then we have

$$
\left(T_{\alpha} x, x\right)-\left(x, T_{\alpha} x\right)=[x, x](\infty)-[x, x](0) .
$$

Since limit-point case holds at $\infty$, one gets $[x, x](\infty)=0$. Further, we obtain from the condition (2.4) that

$$
[x, x](0)=-2 \operatorname{Im} \alpha|x(0)|^{2} .
$$

Substituting (2.6) in (2.5) one gets $\operatorname{Im}\left(T_{\alpha} x, x\right)=\operatorname{Im} \alpha|x(0)|^{2}$ and this completes the proof. 
According to equality (2.6) for $\operatorname{Im} \alpha<0 \quad(\operatorname{Im} \alpha=0$ or $\alpha=\infty) T_{\alpha}$ is an accumulative (self-adjoint) operator in $\mathscr{L}_{r, q}^{2}\left(\mathbf{R}_{q,+}\right)$. Here for $\alpha=\infty$, condition (2.4) should be replaced by $x(0)=0$.

Let us consider the Hilbert space

$$
\mathscr{H}=\mathscr{L}^{2}\left(\mathbf{R}_{-}\right) \oplus \mathscr{L}_{r, q}^{2}\left(\mathbf{R}_{q,+}\right) \oplus \mathscr{L}^{2}\left(\mathbf{R}_{+}\right),
$$

and call it the basic Hilbert space of the dilation.

Let $\operatorname{Dom}\left(\mathscr{M}_{\alpha}\right)$ be the set of all vectors $f=\left\langle\phi_{-}, y, \phi_{+}\right\rangle \in \mathscr{H}$, where $\phi_{-} \in \mathscr{W}_{2}^{1}\left(\mathbf{R}_{-}\right), \phi_{+} \in \mathscr{W}_{2}^{1}\left(\mathbf{R}_{+}\right)$, and $y \in \operatorname{Dom}\left(\mathscr{M}_{\alpha}\right)$ satisfying the conditions

$$
\left(p D_{q^{-1}}\right)(0)-\alpha y(0)=\delta \phi_{-}(0), \quad\left(p D_{q^{-1}}\right)(0)-\bar{\alpha} y(0)=\delta \phi_{+}(0),
$$

where $\delta^{2}:=2 \operatorname{Im} \alpha, \delta>0$.

Let

$$
\mathscr{M}\left\langle\phi_{-}, y, \phi_{+}\right\rangle=\left\langle i \frac{d \phi_{-}}{d \xi}, L y, i \frac{d \phi_{+}}{d \varsigma}\right\rangle
$$

and $\mathscr{M}_{\alpha} f=\mathscr{M} f$ for $f \in \operatorname{Dom}\left(\mathscr{M}_{\alpha}\right)$. Then we have the following assertion.

THEOREM 2.2. The operator $\mathscr{M}_{\alpha}$ is self-adjoint in space $\mathscr{H}$.

Proof. Let $f=\left\langle\phi_{-}, y, \phi_{+}\right\rangle, \quad g=\left\langle\psi_{-}, z, \psi_{+}\right\rangle \in \operatorname{Dom}\left(\mathscr{M}_{\alpha}\right)$. Then with a direct calculation, we have

$$
\begin{aligned}
\left(\mathscr{M}_{\alpha} f, g\right)_{\mathscr{H}} & =\int_{-\infty}^{0} i \phi_{-}^{\prime} \bar{\psi}_{-} d \xi+(L y, z)+\int_{0}^{\infty} i \phi_{+}^{\prime} \bar{\psi}_{+} d \zeta \\
& =i \phi_{-}(0) \bar{\psi}_{-}(0)-i \phi_{+}(0) \bar{\psi}_{+}(0)-[y, z](0)+\left(f, \mathscr{M}_{\alpha} g\right)_{\mathscr{H}} .
\end{aligned}
$$

Using (2.7) one gets that $\left(\mathscr{M}_{\alpha} f, g\right)_{\mathscr{H}}=\left(f, \mathscr{M}_{\alpha} g\right)_{\mathscr{H}}$, i.e. $\mathscr{M}_{\alpha}$ is symmetric.

It can be seen that the operators $\mathscr{M}_{\alpha}$ and $\mathscr{M}_{\alpha}^{*}$ are generated by the same differential expression (2.8). The equality (2.9) can be rewritten as

$$
i \phi_{-}(0) \bar{\psi}_{-}(0)-i \phi_{+}(0) \bar{\psi}_{+}(0)-[y, z](0)=0 .
$$

On the other hand from (2.7) one gets

$$
y(0)=-\frac{i}{\delta}\left(\phi_{+}(0)-\phi_{-}(0)\right), \quad\left(p D_{q^{-1}} y\right)(0)=\delta \phi_{-}(0)-\frac{i \alpha}{\delta}\left(\phi_{+}(0)-\phi_{-}(0)\right) .
$$

Substituting (2.11) in (2.10) we have

$$
\begin{aligned}
i \phi_{-}(0) \bar{\psi}_{-}(0)-i \phi_{+}(0) \bar{\psi}_{+}(0)= & {[y, z](0) } \\
= & -\frac{i}{\delta}\left(\phi_{+}(0)-\phi_{-}(0)\right)\left(p D_{q^{-1}} \bar{z}\right)(0) \\
& -\delta\left[\phi_{-}(0)-\frac{i \alpha}{\delta^{2}}\left(\phi_{+}(0)-\phi_{-}(0)\right)\right] \bar{z}(0) .
\end{aligned}
$$


Since $\phi_{ \pm}(0)$ can be arbitrary numbers, a comparison of the coefficients of $\phi_{ \pm}(0)$ in (2.12) shows that $g=\left\langle\psi_{-}, z, \psi_{+}\right\rangle$satisfies the boundary conditions:

$$
\left(p D_{q^{-1}} z\right)(0)-\alpha z(0)=\delta \psi_{-}(0)
$$

and

$$
\left(p D_{q^{-1}} z\right)(0)-\bar{\alpha} z(0)=\delta \psi_{+}(0) .
$$

This implies that $\mathscr{M}_{\alpha}^{*} \subseteq \mathscr{M}_{\alpha}$, and this completes the proof.

The self-adjoint operator $\mathscr{M}_{\alpha}$ generates in $\mathscr{H}$ a unitary group $\mathscr{Y}_{\alpha}(s)=$ $\exp \left[i \mathscr{M}_{\alpha} s\right](s \in \mathbf{R})$. Denote by $P_{1}: \mathscr{H} \rightarrow \mathscr{L}_{r, q}^{2}\left(\mathbf{R}_{q,+}\right)$ and $P_{2}: \mathscr{L}_{r, q}^{2}\left(\mathbf{R}_{q,+}\right) \rightarrow \mathscr{H}$ the mappings acting according to the formulas $P_{1}:\left\langle\phi_{-}, y, \phi_{+}\right\rangle \rightarrow y$ and $P_{2}: y \rightarrow$ $\langle 0, y, 0\rangle$, respectively. It is known that the operator family $\mathscr{Z}_{\alpha}(s)=P_{1} \mathscr{Y}_{\alpha}(s) P_{2}$ $(s \geq 0)$ is a strongly continuous semigroup of completely nonunitary contraction on $\mathscr{L}_{r, q}^{2}\left(\mathbf{R}_{q,+}\right) \quad([2,13,15,17])$. Let us consider the generator $B_{\alpha}$ of the semigroup $\mathscr{Z}_{\alpha}(s)$ :

$$
B_{\alpha} y=\lim _{s \rightarrow+0}\left[(i s)^{-1}\left(\mathscr{Z}_{\alpha}(s) y-y\right)\right]
$$

where the domain of $B_{\alpha}$ consists of all vectors for which this limit exists. The operator $B_{\alpha}$ is a maximal dissipative operator and further the operator $\mathscr{M}_{\alpha}$ is called the self-adjoint dilation of $B_{\alpha}$.

THEOREM 2.3. The operator $\mathscr{M}_{\alpha}$ is a self-adjoint dilation of $T_{\alpha}$.

Proof. We show that $B_{\alpha}=T_{\alpha}$. Let

$$
\left(\mathscr{M}_{\alpha}-\lambda I\right)^{-1} P_{2} x=g=\left\langle\psi_{-}, y, \psi_{+}\right\rangle,
$$

where $x, y \in \mathscr{L}_{r, q}^{2}\left(\mathbf{R}_{q,+}\right)$ and $\operatorname{Im} \lambda<0$. Then $\left(\mathscr{M}_{\alpha}-\lambda I\right) g=P_{2} x$ and the equation (2.10) is also equivalent to the equation $L y-\lambda y=x$ and

$$
\psi_{-}(\xi)=\psi_{-}(0) e^{-i \lambda \xi}, \quad \psi_{+}(\varsigma)=\psi_{+}(0) e^{-i \lambda \varsigma} .
$$

Since $\psi_{-}$belongs to $\mathscr{L}^{2}\left(\mathbf{R}_{-}\right), \psi_{-}(0)=0$, and $y$ satisfies the boundary condition

$$
\left(p D_{q^{-1}} y\right)(0)-\alpha y(0)=0 .
$$

Moreover, $y \in \operatorname{Dom}\left(T_{\alpha}\right)$, and since a point $\lambda$ with $\operatorname{Im} \lambda<0$ cannot be an eigenvalue of a dissipative operator $T_{\alpha}, y=\left(T_{\alpha}-\lambda I\right)^{-1} x$. Further $\psi_{+}(0)$ can be rewritten as

$$
\psi_{+}(0)=\delta^{-1}\left(\left(p D_{q^{-1}} y\right)(0)-\bar{\alpha} y(0)\right) .
$$

Thus (2.13) is equivalent to

$$
\left(\mathscr{M}_{\alpha}-\lambda I\right)^{-1} P_{2} x=\left\langle 0,\left(T_{\alpha}-\lambda I\right)^{-1} x, \delta^{-1}\left(\left(p D_{q^{-1}} y\right)(0)-\bar{\alpha} y(0)\right) e^{-i \lambda \varsigma}\right\rangle
$$


for $x \in \mathscr{L}_{r, q}^{2}\left(\mathbf{R}_{q,+}\right)$ and $\operatorname{Im} \lambda<0$. Then applying the mapping $P_{1}$ to the last equality we obtain that

$$
P_{1}\left(\mathscr{M}_{\alpha}-\lambda I\right)^{-1} P_{2} x=\left(T_{\alpha}-\lambda I\right)^{-1} x .
$$

On the other hand, for $\operatorname{Im} \lambda<0$, the equality

$$
\begin{aligned}
\left(T_{\alpha}-\lambda I\right)^{-1} & =P_{1}\left(\mathscr{M}_{\alpha}-\lambda I\right)^{-1} P_{2}=-i P_{1} \int_{0}^{\infty} \mathscr{Y}_{\alpha}(s) e^{-i \lambda s} d s P_{2} \\
& =-i \int_{0}^{\infty} \mathscr{Z}_{\alpha}(s) e^{-i \lambda s} d s=\left(B_{\alpha}-\lambda I\right)^{-1} \quad(\operatorname{Im} \lambda<0),
\end{aligned}
$$

holds. (2.14) and (2.15) completes the proof.

\section{Scattering theory of the dilation, functional model and completeness of the root functions of the dissipative operators}

Let us consider two solutions of the equation $(L x)(t)=\lambda x(t)\left(t \in \mathbf{R}_{q,+}\right)$ as $\varphi(t, \lambda)$ and $\psi(t, \lambda)$ that satisfy the conditions

$$
\varphi(0, \lambda)=0, \quad\left(p D_{q^{-1}} \varphi\right)(0, \lambda)=1, \quad \psi(0, \lambda)=1, \quad\left(p D_{q^{-1}} \psi\right)(0, \lambda)=0 .
$$

The Weyl-Titchmarsh function $m_{\infty}$ of the self-adjoint operator $T_{\infty}$ generated by the boundary condition $x(0)=0$ is uniquely determined from the condition

$$
\psi(t, \lambda)+m_{\infty}(\lambda) \varphi(t, \lambda) \in \mathscr{L}_{r, q}^{2}\left(\mathbf{R}_{q,+}\right), \quad \operatorname{Im} \lambda \neq 0 .
$$

It is known that the Weyl-Titchmarsh function $m_{\infty}$ is in general not a meromorphic function on $\mathbf{C}$, but is a holomorphic function with $\operatorname{Im} \lambda \neq 0$, $\operatorname{Im} \lambda \operatorname{Im} m_{\infty}(\lambda)>0$ and it has the property $\overline{m_{\infty}(\lambda)}=m_{\infty}(\bar{\lambda})(\operatorname{Im} \lambda \neq 0)([4,5])$. Further, we let the function $m_{\infty}$ be meromorphic in C. Then $m_{\infty}$ has a countable number of isolated poles on the real axis, these poles are the eigenvalues of the self-adjoint operator $T_{\infty}\left([4,5]\right.$ ), and the operator $T_{\infty}$ (also every self-adjoint extension of the symmetric operator $T_{\min }$ ) has a purely discrete spectrum $([4,5,7,8,16])$.

Due to an important property of the unitary group $\mathscr{Y}_{\alpha}(s)=\exp \left[i \mathscr{M}_{\alpha} s\right]$ $(s \in \mathbf{R})$, we can apply the Lax-Phillips scheme [14] to it. To state more clearly, it has incoming and outgoing subspaces $\mathscr{D}_{-}=\left\langle\mathscr{L}^{2}\left(\mathbf{R}_{-}\right), 0,0\right\rangle$ and $\mathscr{D}_{+}=$ $\left\langle 0,0, \mathscr{L}^{2}\left(\mathbf{R}_{+}\right)\right\rangle$, which satisfy the following properties:

(1) $\mathscr{Y}_{\alpha}(s) \mathscr{D}_{-} \subset \mathscr{D}_{-}, s \leq 0$ and $\mathscr{Y}_{\alpha}(s) \mathscr{D}_{+} \subset \mathscr{D}_{+}, s \geq 0$;

(2) $\bigcap_{s \leq 0} \mathscr{Y}_{\alpha}(s) \mathscr{D}_{-}=\bigcap_{s \geq 0} \mathscr{Y}_{\alpha}(s) \mathscr{D}_{+}=\{0\}$;

(3) $\overline{\bigcup_{s \geq 0} \mathscr{Y}_{\alpha}(s) \mathscr{D}_{-}}=\overline{\bigcup_{s \leq 0} \mathscr{Y}_{\alpha}(s) \mathscr{D}_{+}}=\mathscr{H}$;

(4) $\mathscr{D}_{-} \perp \mathscr{D}_{+}$.

Property (4) is obvious. Let us prove property (1) for $\mathscr{D}_{+}$(the proof for $\mathscr{D}_{-}$ is similar). Set $\mathscr{R}_{\lambda}=\left(\mathscr{M}_{\alpha}-\lambda I\right)^{-1}$. For all $\lambda$ with $\operatorname{Im} \lambda<0$ and for any $f=$ 
$\left\langle 0,0, \varphi_{+}\right\rangle \in \mathscr{D}_{+}$, we have

$$
\mathscr{R}_{\lambda} f=\left\langle 0,0,-i e^{-i \lambda \xi} \int_{0}^{\xi} e^{i \lambda s} \varphi_{+}(s) d s\right\rangle .
$$

So we have $\mathscr{R}_{\lambda} f \in \mathscr{D}_{+}$. Hence for $g \perp \mathscr{D}_{+}$, we get that

$$
\left(\mathscr{R}_{\lambda} f, g\right)_{\mathscr{H}}=-i \int_{0}^{\infty} e^{-i \lambda s}\left(\mathscr{Y}_{\alpha}(s) f, g\right)_{\mathscr{H}} d s=0, \quad \operatorname{Im} \lambda<0
$$

and hence $\left(\mathscr{Y}_{\alpha}(s) f, g\right)_{\mathscr{H}}=0$ for all $s \geq 0$. This implies that $\mathscr{Y}_{\alpha}(s) \mathscr{D}_{+} \subset \mathscr{D}_{+}$for $s \geq 0$, and therefore (1) is proved.

It is known that the generator of the semigroup $\mathscr{V}(s)$ of the one-sided shift in the space $\mathscr{L}^{2}\left(\mathbf{R}_{+}\right)$is the differential operator $i \frac{d}{d \xi}$ with the boundary condition $\varphi(0)=0$. Now let us define the semigroup of isometries $\mathscr{Y}_{\alpha}^{+}(s)=\mathscr{P}_{1}^{+} \mathscr{Y}_{\alpha}(s) P_{1}^{+}$, $s \geq 0$, where $\mathscr{P}_{1}^{+}: \mathscr{H} \rightarrow \mathscr{L}^{2}\left(\mathbf{R}_{+}\right)$and $\mathscr{P}_{2}^{+}: \mathscr{L}^{2}\left(\mathbf{R}_{+}\right) \rightarrow \mathscr{D}_{+}\left(\mathscr{P}_{1}^{+}:\left\langle\phi_{-}, y, \phi_{+}\right\rangle \rightarrow\right.$ $\left.\phi_{+}, \mathscr{P}_{2}^{+}: \phi \rightarrow\langle 0,0, \phi\rangle\right)$. On the other side the generator $S$ of the semigroup of isometries $\mathscr{Y}_{\alpha}^{+}(s)$ in $\mathscr{L}^{2}\left(\mathbf{R}_{+}\right)$, is the operator

$$
S \phi=\mathscr{P}_{1}^{+} \mathscr{M}_{\alpha} \mathscr{P}_{2}^{+} \phi=\mathscr{P}_{1}^{+} \mathscr{M}_{\alpha}\langle 0,0, \phi\rangle=\mathscr{P}_{1}^{+}\left\langle 0,0, i \frac{d \phi}{d \xi}\right\rangle=i \frac{d \phi}{d \xi},
$$

where $\phi \in \mathscr{W}_{2}^{1}\left(\mathbf{R}_{+}\right)$and $\phi(0)=0$. Since a semigroup is uniquely determined by its generator, we get $\mathscr{Y}_{\alpha}^{+}(s)=\mathscr{V}(s)$, and so,

$$
\bigcap_{s \geq 0} \mathscr{Y}_{\alpha}(s) \mathscr{D}_{+}=\left\langle 0,0, \bigcap_{s \geq 0} \mathscr{V}(s) \mathscr{L}^{2}\left(\mathbf{R}_{+}\right)\right\rangle=\{0\},
$$

i.e., property (2) is proved.

As stated in the scheme of the Lax-Phillips scattering theory, the scattering matrix is defined using the spectral representations theory. Now, we shall continue with their construction. During this process, we will have proved property (3) of the incoming and outgoing subspaces.

We first prove the following lemma.

LEMMA 3.1. The operator $T_{\alpha}$ is completely non-self-adjoint (pure).

Proof. Let us assume the contrary. Let $H^{\prime} \subset \mathscr{L}_{r, q}^{2}\left(\mathbf{R}_{q,+}\right)$ be a nontrivial subspace of $\mathscr{L}_{r, q}^{2}\left(\mathbf{R}_{q,+}\right)$ in which $T_{\alpha}$ has a self-adjoint part $T_{\alpha}^{\prime}$ in it. If $x \in \operatorname{Dom}\left(T_{\alpha}^{\prime}\right)$, then $x \in \operatorname{Dom}\left(T_{\alpha}^{*}\right)$, and

$$
\left(p D_{q^{-1}} x\right)(0)-\alpha x(0)=0, \quad\left(p D_{q^{-1}} x\right)(0)-\bar{\alpha} x(0)=0 .
$$

From this discussion, for the eigenfunctions $x(t, \lambda)$ of the operator $L_{\alpha}$ that lie in $H^{\prime}$ and are eigenvectors of $L_{\alpha}^{\prime}$ we have

$$
x(0, \lambda)=0, \quad\left(p D_{q^{-1}} x\right)(0, \lambda)=0,
$$


and then by the uniqueness theorem of the Cauchy problem for the equation $(L x)(t)=\lambda x(t),\left(t \in \mathbf{R}_{q,+}\right)$, we have $x(t, \lambda) \equiv 0$. Since $m_{\infty}(\lambda)$ is a meromorphic function in $\mathbf{C}$, it can be concluded that the resolvent $\mathscr{R}_{\lambda}\left(T_{\alpha}\right)$ of the operator $T_{\alpha}$ is a compact operator, and hence the spectrum of $T_{\alpha}^{\prime}$ is purely discrete. Hence by the theorem on expansion in eigenfunctions of the self-adjoint operator $T_{\alpha}^{\prime}$, we have $H^{\prime}=\{0\}$, i.e., the operator $T_{\alpha}$ is simple. The lemma is proved.

Passing to the proof of property (3), we let

$$
\mathscr{K}_{-}=\overline{\bigcup_{s \geq 0} \mathscr{Y}_{\alpha}(s) \mathscr{D}_{-}}, \quad \mathscr{K}_{+}=\overline{\bigcup_{s \leq 0} \mathscr{Y}_{\alpha}(s) \mathscr{D}_{+}} .
$$

Lemma 3.2. The equality $\mathscr{K}_{-}+\mathscr{K}_{+}=\mathscr{H}$ holds.

Proof. Indeed, by taking into account the properties (1) of the subspaces $\mathscr{D}_{ \pm}$it is easy to see that the subspace $\mathscr{H}^{\prime}=\mathscr{H} \ominus\left(\mathscr{K}_{-}+\mathscr{K}_{+}\right)$is invariant under the group $\left\{\mathscr{Y}_{\alpha}(s)\right\}$ and has the form $\mathscr{H}^{\prime}=\left\langle 0, H^{\prime}, 0\right\rangle$, where $H^{\prime}$ is a subspace of $\mathscr{L}_{r, q}^{2}\left(\mathbf{R}_{q,+}\right)$. Therefore, if the subspace $\mathscr{H}^{\prime}$ (and hence also $H^{\prime}$ ) were nontrivial, then the unitary group $\left\{\mathscr{Y}_{\alpha}^{\prime}(s)\right\}$, restricted to this subspace, would be a unitary part of the group $\left\{\mathscr{Y}_{\alpha}(s)\right\}$, and hence the restriction of $T_{\alpha}$ to $H^{\prime}$ would be a selfadjoint operator acting in $H^{\prime}$. But that is excluded by virtue of Lemma 3.1. The lemma is proved.

Let us adopt the following notations: $\omega(x, \lambda):=\psi(x, \lambda)+m_{\infty}(\lambda) \varphi(x, \lambda)$,

$$
\Theta_{\alpha}(\lambda):=\frac{m_{\infty}(\lambda)-\alpha}{m_{\infty}(\lambda)-\bar{\alpha}} .
$$

Now consider the vectors $\Psi_{\lambda}^{\mp}(x, \xi, \varsigma)$ as

$$
\Psi_{\lambda}^{-}(x, \xi, \varsigma)=\left\langle e^{-i \lambda \xi},\left(m_{\infty}(\lambda)-\alpha\right)^{-1} \delta \omega(x, \lambda), \overline{\Theta_{\alpha}}(\lambda) e^{-i \lambda \varsigma}\right\rangle
$$

and

$$
\Psi_{\lambda}^{+}(x, \xi, \varsigma)=\left\langle\Theta_{\alpha}(\lambda) e^{-i \lambda \xi},\left(m_{\infty}(\lambda)-\bar{\alpha}\right)^{-1} \delta \omega(x, \lambda), e^{-i \lambda \varsigma}\right\rangle .
$$

These vectors do not belong to the space $\mathscr{H}$ for real $\lambda$. But they satisfy the equation $\mathscr{M} \Psi=\lambda \Psi$ and boundary conditions for $\mathscr{M}_{\alpha}$.

Let $\Phi_{\mp}: f \rightarrow \tilde{f}_{\mp}(\lambda)$, where

$$
\left(\Phi_{\mp} f\right)(\lambda)=\tilde{f}_{\mp}(\lambda)=\frac{1}{\sqrt{2 \pi}}\left(f, \Psi_{\lambda}^{\mp}\right)_{\mathscr{H}},
$$

$f=\left\{\phi_{-}, y, \phi_{+}\right\}$, and $\phi_{-}, y$ and $\phi_{+}$are smooth, compactly supported functions. For $f=\left\{\phi_{-}, 0,0\right\}, g=\left\{\chi_{-}, 0,0\right\} \in \mathscr{D}_{-}$, the equality

$$
\tilde{f}_{-}(\lambda)=\frac{1}{\sqrt{2 \pi}}\left(f, \Psi_{\lambda}^{-}\right)_{\mathscr{H}}=\frac{1}{\sqrt{2 \pi}} \int_{-\infty}^{0} \phi_{-}(s) \exp (i \lambda s) d s
$$


holds. Hence $\tilde{f}_{-}(\lambda)$ belongs to $\mathscr{H}_{-}^{2}$. Now let us consider the dense set $\tilde{\mathscr{K}}_{-}$in $\mathscr{K}_{-}$consisting of all vectors $f$ such that $f$ is a compactly supported function in $\mathscr{D}_{-}$and $f \in \tilde{\mathscr{K}}_{-}$if $f=\mathscr{Y}_{\alpha}(s) f_{0}, f_{0}=\left\{\phi_{-}, 0,0\right\}, \phi_{-} \in C_{0}^{\infty}\left(\mathbf{R}_{-}\right)$(where $C_{0}^{\infty}\left(\mathbf{R}_{-}\right.$) denotes the set of all smooth, compactly supported functions defined on $\mathbf{R}_{-}$, and $s=s_{f}$ is a nonnegative number). Then if $f, g \in \mathscr{K}_{-}$we have for $s>s_{f}$ and $s>s_{g}$ that $\mathscr{Y}_{\alpha}(-s) f, \mathscr{Y}_{\alpha}(-s) g \in \mathscr{D}_{-}$and their first component belong to $C_{0}^{\infty}\left(\mathbf{R}_{-}\right)$. Therefore

$$
\begin{aligned}
(f, g)_{\mathscr{H}} & =\left(\mathscr{Y}_{\alpha}(-s) f, \mathscr{Y}_{\alpha}(-s) g\right)_{\mathscr{H}}=\left(\Phi_{-} \mathscr{Y}_{\alpha}(-s) f, \Phi_{-} \mathscr{Y}_{\alpha}(-s) g\right)_{\mathscr{L}^{2}} \\
& =\left(\exp (-i \lambda s) \mathscr{Y}_{\alpha}(-s) f, \exp (-i \lambda s) \mathscr{Y}_{\alpha}(-s) g\right)_{\mathscr{L}^{2}}=\left(\Phi_{-} f, \Phi_{-} g\right)_{\mathscr{L}^{2}} .
\end{aligned}
$$

Taking closure in (3.5) we obtain Parseval equality for the space $\mathscr{K}_{-}$. Further the inversion formula

$$
f=\frac{1}{\sqrt{2 \pi}} \int_{-\infty}^{\infty} \tilde{f}_{-}(\lambda) \overline{\Psi_{\lambda}^{-}} d \lambda
$$

follows from the Parseval equality if all integrals are taken as limits in the mean of the intervals. Finally we have

$$
\Phi_{-} \mathscr{K}_{-}=\overline{\bigcup_{s \geq 0} \Phi_{-} \mathscr{Y}_{\alpha}(s) \mathscr{D}_{-}}=\overline{\bigcup_{s \geq 0} \exp (-i \lambda s) \mathscr{H}_{-}^{2}}=\mathscr{L}^{2}(\mathbf{R}) .
$$

This implies that $\mathscr{K}_{-}$is isometrically identical with $\mathscr{L}^{2}(\mathbf{R})$. Similarly one can show that $\mathscr{K}_{+}$is isometrically identical with $\mathscr{L}^{2}(\mathbf{R})$.

According to (3.2), the function $\Theta_{\alpha}$ satisfies $\left|\Theta_{\alpha}(\lambda)\right|=1$ for $\lambda \in \mathbf{R}$. Therefore, it follows from the explicit formulas for the vectors $\Psi_{\lambda}^{+}$and $\Psi_{\lambda}^{-}$that

$$
\Psi_{\lambda}^{-}=\bar{\Theta}_{\alpha}(\lambda) \Psi_{\lambda}^{+} \quad(\lambda \in \mathbf{R}) .
$$

Moreover $\mathscr{K}_{-}=\mathscr{K}_{+}$. Together with Lemma 3.2 , this shows that $\mathscr{H}=\mathscr{K}_{-}=$ $\mathscr{K}_{+}$, and property (3) above has been established for the incoming and outgoing subspaces.

Hence, $\Phi_{-}$isometrically maps onto $\mathscr{L}^{2}(\mathbf{R})$ with the subspace $\mathscr{D}_{-}$mapped onto $\mathscr{H}_{-}^{2}$, and the operators $\mathscr{Y}_{\alpha}(s)$ are transformed by the operators of multiplication by $e^{i \lambda s}$. This means that $\Phi_{-}\left(\Phi_{+}\right)$is the incoming (outgoing) spectral representation for the group $\left\{\mathscr{Y}_{\alpha}(s)\right\}$. Using (3.6), we can pass from the $\Phi_{+}-$ representation of a vector $f \in \mathscr{H}$ to its $\Phi_{-}$-representation by multiplication of the function $\Theta_{\alpha}(\lambda): \tilde{f}_{-}(\lambda)=\Theta_{\alpha}(\lambda) \tilde{f}_{+}(\lambda)$. Based on [14], the scattering function of the group $\left\{\mathscr{Y}_{\alpha}(s)\right\}$ with respect to the subspaces $\mathscr{D}_{-}$and $\mathscr{D}_{+}$, is the coefficient by which the $\Phi_{-}$-representation of a vector $f_{\tilde{f}} \in \mathscr{H}$ must be multiplied in order to get the corresponding $\Phi_{+}$-representation: $\tilde{f}_{+}(\lambda)=\bar{\Theta}_{\alpha}(\lambda) \tilde{f}_{-}(\lambda)$, and thus we have proved the following statement.

THEOREM 3.3. The function $\bar{\Theta}_{\alpha}$ is the scattering function of the group $\left\{\mathscr{Y}_{\alpha}(s)\right\}$ (of the self-adjoint operator $\mathscr{M}_{\alpha}$ ). 
Let $\mathscr{K}=\left\langle 0, \mathscr{L}_{r, q}^{2}\left(\mathbf{R}_{q,+}\right), 0\right\rangle$, so that $\mathscr{H}=\mathscr{D}_{-} \oplus \mathscr{K} \oplus \mathscr{D}_{+}$. It follows from the explicit form of the unitary transformation $\Phi_{-}$that under the mapping $\Phi_{-}$

$$
\mathscr{H} \rightarrow \mathscr{L}^{2}(\mathbf{R}), \quad f \rightarrow \tilde{f}_{-}(\lambda)=\left(\Phi_{-} f\right)(\lambda), \quad \mathscr{D}_{-} \rightarrow \mathscr{H}_{-}^{2}, \quad \mathscr{D}_{+} \rightarrow \Theta_{\alpha^{\prime}} \mathscr{H}_{+}^{2}
$$

and

$$
\mathscr{K} \rightarrow \mathscr{H}_{+}^{2} \ominus \Theta_{\alpha} \mathscr{H}_{+}^{2}, \quad \mathscr{Y}_{\alpha}(s) f \rightarrow\left(\Phi_{-} \mathscr{Y}_{\alpha}(s) \Phi_{-}^{-1} \tilde{f}_{-}\right)(\lambda)=e^{i \lambda s} \tilde{f}_{-}(\lambda)
$$

The formulas (3.7)-(3.8) show that our operator $T_{\alpha}$ is a unitary equivalent to the model dissipative operator with the characteristic function $\Theta_{\alpha}$. Since the characteristic functions of unitary equivalent dissipative operators coincide (see $[1,2$, $13,15,17,18])$, we have proved the next theorem.

THEOREM 3.4. The characteristic function of the dissipative operator $T_{\alpha}$ coincides with the function $\Theta_{\alpha}$ defined in (3.2).

It is known that the characteristic function of a dissipative operator carries complete information about the spectral properties of this operator (see $[1,2$, $13,15,17,18])$. For example, the absence of a singular factor $s_{\alpha}(\lambda)$ of the characteristic function $\Theta_{\alpha}$ in the factorization $\Theta_{\alpha}(\lambda)=s_{\alpha}(\lambda) \mathscr{B}_{\alpha}(\lambda)$ (where $\mathscr{B}_{\alpha}(\lambda)$ is a Blaschke product $[15,17])$ guarantees the completeness of the system of eigenfunctions and associated functions (or root functions) of the dissipative $q$-Sturm-Liouville operator $T_{\alpha}([1,2,13,15,17,18])$.

THEOREM 3.5. Let the function $m_{\infty}$ be meromorphic in $\mathbf{C}$. Then, for all values of $\alpha$ with $\operatorname{Im} \alpha>0$, except possibly for a single value $\alpha=\alpha_{0}$, the characteristic function $\Theta_{\alpha}$ of the dissipative operator $T_{\alpha}$ is a Blaschke product, and the spectrum of $T_{\alpha}$ is purely discrete and belongs to the open upper half-plane. The operator $T_{\alpha}\left(\alpha \neq \alpha_{0}\right)$ has a countable number of isolated eigenvalues with finite multiplicity and limit points at infinity, and the system of all eigenfunctions and associated functions (or root functions) of this operator is complete in the space $\mathrm{L}_{r, q}^{2}\left(\mathbf{R}_{q,+}\right)$.

Proof. We have $\operatorname{Im} \lambda \operatorname{Im} m_{\infty}(\lambda)>0$ for all $\operatorname{Im} \lambda \neq 0$, and $\overline{m_{\infty}(\lambda)}=m_{\infty}(\bar{\lambda})$ for all $\lambda \in \mathbf{C}$, except the real poles of $m_{\infty}(\lambda)$. Thus, it follows from (3.2) that $\left|\Theta_{\alpha}(\lambda)\right| \leq 1$ for all $\lambda \in \mathbf{C}_{+}$and $\left|\Theta_{\alpha}(\lambda)\right|=1$ for almost all $\lambda \in \mathbf{R}$, i.e., $\Theta_{\alpha}(\lambda)$ is an inner function in the upper half-plane, and it is meromorphic in the whole complex $\lambda$-plane. Therefore, it can be factored as follows

$$
\Theta_{\alpha}(\lambda)=e^{i \lambda d} \mathscr{B}_{\alpha}(\lambda), \quad d=d(\alpha) \geq 0,
$$

where $\mathscr{B}_{\alpha}(\lambda)$ is a Blaschke product. Thus, we find that

$$
\left|\Theta_{\alpha}(\lambda)\right|=\left|e^{i \lambda d}\right|\left|\mathscr{B}_{\alpha}(\lambda)\right| \leq e^{-d(\alpha) \operatorname{Im} \lambda}, \quad \operatorname{Im} \lambda \geq 0 .
$$


Further, for $m_{\infty}(\lambda)$ in terms of $\Theta_{\alpha}(\lambda),(3.2)$ gives us that

$$
m_{\infty}(\lambda)=\frac{\bar{\alpha} \Theta_{\alpha}(\lambda)-\alpha}{\Theta_{\alpha}(\lambda)-1} .
$$

If $d(\alpha)>0$ for a given value $\alpha(\operatorname{Im} \alpha>0)$, then by (3.10) we have

$$
\lim _{s \rightarrow+\infty} \Theta_{\alpha}(i s)=0
$$

which together with (3.11) implies that

$$
\lim _{s \rightarrow+\infty} m_{\infty}(i s)=-\alpha .
$$

Since $m_{\infty}(\lambda)$ is independent of $\alpha, d(\alpha)$ can be nonzero at not more than a single point $\alpha=\alpha_{0}$ and, further

$$
\alpha_{0}=-\lim _{s \rightarrow+\infty} m_{\infty}(i s)
$$

The theorem is proved.

It should be noted that all results obtained for dissipative operators can be immediately transferred to accumulative operators, because a linear operator $\mathbf{S}$ acting in a Hilbert space $\mathbf{H}$ is accumulative if and only if $-\mathbf{S}$ is dissipative. Then Theorem 3.5 yields the following result.

COROllary 3.6. Let the function $m_{\infty}$ be meromorphic in $\mathbf{C}$. Then, for all values of $\alpha$ with $\operatorname{Im} \alpha<0$, except possibly for a single value $\alpha=\alpha_{1}$, the spectrum of accumulative operator $T_{\alpha}$ is purely discrete and belongs to the open lower halfplane. The operator $T_{\alpha}\left(\alpha \neq \alpha_{1}\right)$ has a countable number of isolated eigenvalues with finite multiplicity and limit points at infinity, and the system of all eigenfunctions and associated functions (or root functions) of this operator is complete in the space $\mathrm{L}_{r, q}^{2}\left(\mathbf{R}_{q,+}\right)$.

\section{REFERENCES}

[1] B. P. Allahverdiev, Dissipative Sturm-Liouville operators in limit-point case, Acta Appl. Math. 86 (2005), 237-248.

[2] B. P. Allahverdiev, Nonself-adjoint Sturm-Liouville operators in limit-circle case, Taiwan. J. Math. 16 (2012), 2035-2052.

[ 3 ] M. H. AnNaby and Z. S. Mansour, $q$-fractional calculus and equations, Lecture notes in math. 2056, Springer, Heidelberg, 2012.

[4] M. H. Annaby, H. A. Hassan and Z. S. Mansour, Sampling theorems associated with singular $q$-Sturm Liouville problems, Result. Math. 62 (2012), 121-136.

[5] M. H. Annaby, Z. S. Mansour and I. A. Soliman, $q$-Titchmarsh-Weyl theory: series expansion, Nagoya Math. J. 205 (2012), 67-118.

[6] M. H. Annaby and Z. S. Mansour, Basic Sturm-Liouville problems, Phys. A. Math. Gen. 38 (2005), 3775-3797. 
[7] F. V. Atkinson, Discrete and continuous boundary problems, Academic Press, New York, 1964.

[ 8 ] N. Dunford AND J. T. Schwartz, Linear operators, Part II, Interscience, New York, 1964.

[9] T. ERnst, The History of $q$-calculus and a new method, Uppsala, 2000.

[10] H. ExтоN, $q$-hipergeometric functons and applications, Ellis-Horwood, Chichester, 1983.

[11] W. Hahn, Beiträge zur Theorie der Heineschen Reihen, Math. Nachr. 2 (1949), 340-379 (in German).

[12] F. H. JACKson, On q-definite integrals, Quart. J. Pure Appl. Math. 41 (1910), 193-203.

[13] A. KuzHEL, Characteristic functions and models of nonself-adjoint operators, Kluwer Acad. Publ., Dordrecht, 1996.

[14] P. D. Lax ANd R. S. Phillips, Scattering theory, Academic Press, New York, 1967.

[15] B. Sz.-Nagy And C. Folaş, Analyse harmonique des operateurs de l'espace de Hilbert, Masson, Paris; Akad. Kiadó, Budapest, 1967 (in French), North-Holland, Amsterdam; Akad. Kiadó, Budapest, 1970 (English translation).

[16] M. A. Naimark, Linear differential operators, 2nd ed, Moscow, Nauka, 1969 (in Russian), English transl. of 1st ed, Parts 1, 2, Ungar, New York, 1967, 1968.

[17] B. S. Pavlov, Spectral analysis of a dissipative singular Schrödinger operator in terms of a functional model, Itogi Nauki Tekh. Ser. Sovrem. Probl. Mat. Fundam. Napravleniya 65 (1991), 95-163, Partial Differential Equations VIII, Encyc. Math. Sci. 65 (1996), 87-153 (English translation).

[18] B. S. Pavlov, Irreversibility, Lax-Phillips approach to resonance scattering and spectral analysis of non-self-adjoint operators in Hilbert space, Int. J. Theor. Phys. 38 (1999), 21-45.

Bilender P. Allahverdiev

Department of Mathematics

SulEyMaN DEMIREL UNIVERSITY

32260 ISPARTA

TURKEY

E-mail: bilenderpasaoglu@sdu.edu.tr 